\title{
Active Galactic Nuclei and gamma rays
}

\author{
Berrie Giebels \\ Laboratoire Leprince-Ringuet, Ecole polytechnique \\ 91128 Palaiseau, France \\ E-mail: berrie@in2p3.fr \\ Felix Aharonian \\ Max-Planck-Institut für Kernphysik \\ 69029 Heidelberg, Germany \\ E-mail: Felix.Aharonian@mpi-hd.mpg.de \\ Hélène Sol \\ LUTH, Observatoire de Paris \\ 5 place Jules Janssen, 92190 Meudon, France \\ E-mail: Helene.Sol@obspm.fr
}

\begin{abstract}
The supermassive black holes harboured in active galactic nuclei are at the origin of powerful jets which can emit copious amounts of $\gamma$-rays. The exact interplay between the infalling matter, the black hole and the relativistic outflow is still poorly known, and this parallel session of the $12^{\text {th }}$ Marcel Grossman meeting intended to offer the most up to date status of observational results with the latest generation of ground and space-based instruments, as well as the theoretical developments relevant for the field.
\end{abstract}

Keywords: Style file; LATEX; Proceedings; World Scientific Publishing.

\section{Observational context}

The detection of cosmic $\gamma$-rays is essentially based on either direct or indirect methods. The former uses satellised high-energy particle physics type detectors, based on the tracking of the $\gamma$-ray pair conversion and the calorimetry of the subsequent electromagnetic cascade. This technique is constrained almost by definition in weight, size and power consumption, which limits respectively the maximal $\gamma$-ray energy, the effective area, and the dynamic range. They have however a large field of view (fov), almost avoiding any need for pointed observations since the performance of the instrument (angular and energy resolution) changes slowly within the fov. With livetimes close to $90 \%$, space-based observatories provide some of the best continuous monitoring of the high-energy sky ever realized.

The indirect methods rely on the detection of the secondary particles induced by the $\gamma$-ray pair creation in the atmosphere. This can be done directly on the ground, with large area all-weather water Čerenkov detectors or air shower detectors (such as MILAGRO and ARGO-YBJ) at the price of $\sim \mathrm{TeV}$ energy thresholds and poor background rejection. Another technique consist in the indirect detection of the air cascade at energies $\geq 10 \mathrm{GeV}$, using the faint optical Cerenkov flash of the shower with atmospheric Cerenkov telescopes (ACTs), with the drawback of having a limited livetime $\sim 10 \%$ due to the same constraint as ground-based optical telescopes of clear, dark night skies for carrying out observations limiting light 
curves to be continuous only over a time up to $\sim 6 \mathrm{~h}$, with varying sensitivities and energy thresholds due to the specifics of this technique. Since such cascades, or even the Cerenkov light pool, have transverse sizes $o(100 \mathrm{~m})$, the effective area can be much larger than the physical size of the detector itself, and, provided an efficient hadronic background, have a tremendous sensitivity. Clearly, space- and groundbased instruments are extremely complementary, and are very likely to remain so until much larger $\gamma$-ray detectors can be put in space.

\subsection{Space missions}

After a long generation of $\gamma$-ray detectors based on moderately precise sparkchambers technology running on expandable gas, such as SAS-2 (1972-1973), COS-B (1975-1982) and EGRET (on board of CGRO, 1991-2000), the newest and most recent observatories AGILE and Fermi-LAT use robust and finely segmented siliconbased trackers, improving the angular resolution by an order of magnitudea but also increasing the fov up to about $\pi$ sr through a more compact design. the LAT onboar Fermi detects $\gamma$-rays in the range $20 \mathrm{MeV}-300 \mathrm{GeV}$ where the lowest energy is limited by the background and the highest energy mostly by statistics. The whole sky is covered within two orbits, or $3 \mathrm{~h}$, with an integrated flux sensitivity above $100 \mathrm{MeV}$ of $\sim 2 \times 10^{-6} \mathrm{~cm}^{-2} \mathrm{~s}^{-1}$ and $\sim 4 \times 10^{-7} \mathrm{~cm}^{-2} \mathrm{~s}^{-1}$ for respectively Galactic and extragalactic ones, providing among the longest light curves ever recorded ${ }^{3}$. An extremely important figure is that the LAT detects the Crab nebula, a standard candle used in $\mathrm{TeV}$ astronomy, within a year at $\sim 100 \mathrm{GeV}$, an energy usually defined as the border between high energy (HE, $E>100 \mathrm{MeV}$ ) and very high energy (VHE, $E>100 \mathrm{GeV}$ ) where ACTs become sensitive. Of crucial importance for AGN studies evoked here is of course the LAT's uniform all-sky monitoring capabilities along with its efficiency, allowing observers at any other wavelength to have (almost) guaranteed $\mathrm{GeV}$ observations of their favourite sources without need to apply for observations. Within a year, the LAT has collected $\sim 200 \times 10^{6} \gamma$-rays, 2 orders of magnitude more than its predecessor. The first Fermi catalog ${ }^{2}$ (1FGL), based on 11 months of scientific operations, contains 1451 sources of which 1043 are at Galactic latitudes $b>10^{\circ}$. From this latter sample was then derived the first LAT AGN catalog (1LAC), comprising 300 BL Lac objects (compared to 13 for EGRET), 296 flat-spectrum radio quasars (FSRQs, of which EGRET saw 66), and 113 AGN of other (or unknown) type. This is a tremendous change of the HE sky with just one of the 10 expected years of scientific operations, so even more is to be expected in the future.

$\begin{array}{lllll}{ }^{\mathrm{a}} \text { For } & \text { a } & \text { summary } & \text { of } & \text { the }\end{array}$ http://www-glast.slac.stanford.edu/software/IS/glast_lat_performance.htm 


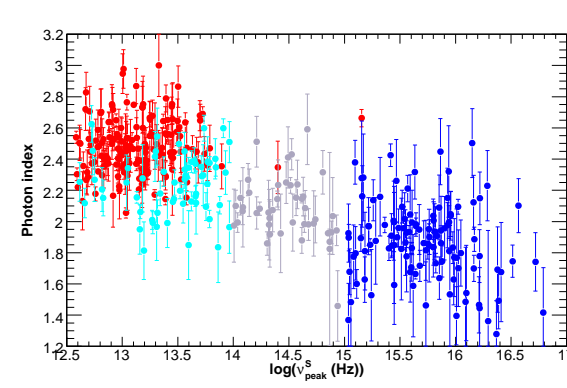

(a)

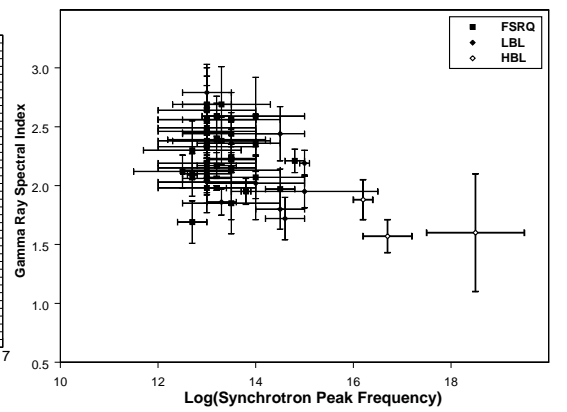

(b)

Fig. 1. (a) The Fermi blazar photon index as a function of its derived synchrotron peak frequency $\nu_{\text {peak }}^{\mathrm{s}}$, as derived in Ref. 4] (b) A similar study using the EGRET data, as published in Ref. [5. Thanks to a much larger population and a better energy resolution, a clear correlation appears where the blazar spectrum becomes increasingly harder when the synchrotron peak $\nu_{\text {peak }}^{\mathrm{s}}$ moves blueward from the optical to the X-rays.

\subsection{Ground-based telescopes}

The most sensitive extensive air shower (EAS) detector to date is the Milagro observatory, which has provided a 1 to $100 \mathrm{TeV}$ survey of the $\gamma$-ray sky in which 34 galactic Fermi sources were found 9 ? So far however ACTs have been the dominant technique for studying the VHE extragalactic field, since the $\gamma$-ray horizon at $\mathrm{TeV}$ energies is (at best) located at $z \sim 0.1$ due to photon-photon interactions with the extragalactic background light (EBL).

The new generation of major ACTs (HESS, MAGIC and VERITAS mainly), with larger telescopes and improved reconstruction techniques, have augmented the flux sensitivity of this technique by an order of magnitude within a decade, reaching a significance of 5 standard deviations on Crab nebula type sources in $\sim 30$ s or less, and ways are found to decrease the effect of moonlight in order to increase the observation time. The population of extragalactic "TeV sources" is currently at 30 , but continuously increasing as can be seen in Fig. 2. Most of the sources are BL Lacs with fluxes at the level of a few percent of the Crab nebula equivalent, with little measurable variability.

\section{Gamma-ray AGN}

While it is widely accepted that blazar $\gamma$-ray emission originates from inverse Compton emission in an ultrarelativistic jet pointing within a few degrees of our line of sight, it is still not clear where in the jet this emission occurs, nor what the source of the scattered soft photons is. Beyond these questions, it has also yet to be established

${ }^{\mathrm{b}}$ See the catalog TeVCat http://tevcat.in2p3.fr 


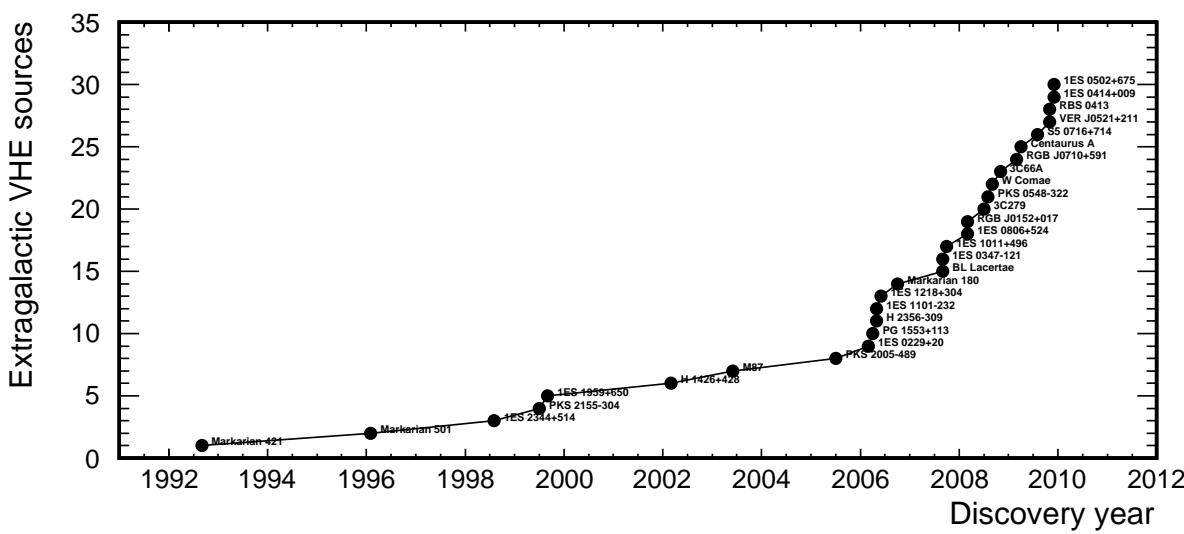

Fig. 2. The number of extragalactic sources detected by ACTs, as a funcion of their detection date, using the TeVCaT. The slope change from $\sim 0.5 /$ year to $\sim 5 /$ year coincides with the current generation of ACTs becoming operational, illustrating the dramatic effect of the sensitivity increase.

what role the central black hole exactly plays in the mechanism that powers the jet. The extremely efficient release of binding energy of accreting gas, as well as directly extracting spin energy from the black hole through the Blandford-Znajek mechanism 11 , are possible. The newly available means to perform $\gamma$-ray multi-wavelength monitoring of the nonthermal emission will certainly provide clues for a better understanding about the jet composition, and perhaps about jet formation as well. The spectral energy distribution (SED) of blazars in $\nu F_{\nu}$ representation usually has two broad components, with the lower one generally believed to be synchrotron radiation, and the higher one inverse Compton (IC) radiation from the same electrons on target photons which can have different origins.

At the time this review is being written, all but 4 of the grand ensemble of extragalactic $\gamma$-ray sky sources are seen by Fermi, so the global HE spectral characteristics can be well summarized by the 1LAC sample. Fig. 1(a) shows that the HE $\gamma$-ray index $\Gamma_{\mathrm{HE}}$ becomes harder when the synchrotron peak shifts globally blueward. This should be compared with Fig. 2 in Ref. 5, displayed in Fig. 1(b), where this trend was first seen using only EGRET blazars, but with 37 FSRQs and 13 BL Lacs. It appears hence that the FSRQ $\gamma$-ray spectra are different from the BL Lac population, with the average photon index of the former being $\geq 2.2$, while the average photon index of the latter is $<2$. The blazar index versus luminosity characteristics as seen by Fermi appear also to largely follow the so-called blazar sequence $e^{\frac{15 \mid 16}{16}}$ based on a one zone, homogeneous synchrotron self-Compton and external Compton model, where the peak luminosities of the two radiative components are located at increasingly higher frequencies when the observed $\gamma$-ray luminosity decreases, and where the external Compton cooling becomes progressively more inefficient than the self-Compton. 
On the other side, the observed VHE spectra from all extragalactic objects have photon indexes $>2$, so the combination of Fermi and ACTs constrains for almost all BL Lacs the peak of the observed SED (in $\nu F_{\nu}$ representation) 10 . This should now allow the best characterization ever made of both components of the SED, the monitoring with unprecedented accuracy of how these components evolve, and how they are related.

\subsection{FSRQ}

The FSRQ class of blazars are the most powerful $\gamma$-ray emitters as well as the most luminous. This is best illustrated by the gigantic flare ${ }^{14}$ exhibited by $3 \mathrm{C}$ 454.3 on December 2, 2009, when it reached a flux of $F(E>100 \mathrm{MeV}) \simeq 2 \times$ $10^{-5} \mathrm{~cm}^{-2} \mathrm{~s}^{-1}$, which is up to date the brightest transient ever detected in the HE range. For comparison, the brightest persistent source in the HE sky is the Vela pulsar, at about half that flux. This population is also extremely variable, and their spectra show significant spectral curvature in the HE range. Since Fermi probes these sources mostly at their peak luminosity frequency, and with unprecedented resolution, theorists working on FSRQs are guaranteed to have a field day. The detection of the FSRQ 3C279 $\frac{18}{18}$ at $z=0.536$ during an optical flare in 2006 by the MAGIC experiment at the $5.8 \sigma$ level and a dedicated low-energy analysis was the first of this kind with an ACT. The corresponding GeV flux, had Fermi been there to see it, would have been a really exceptional state, an order of magnitude higher than the highest $\mathrm{GeV}$ flux seen so far on this source, as is pointed out in Ref. 10 in order to match the Fermi extrapolation to higher energies assuming a conservative EBL attenuation and no further spectral steepening. This detection is clearly paving the way for upcoming ACTs with $<100 \mathrm{GeV}$ thresholds, which could probe the FSRQ Compton component variability at timescales not accessible to Fermi, and eventually find out whether they can exhibit very fast variability as well.

\subsection{BL Lacertae}

The most constraining observations on jet kinematics and the $\gamma$-ray emitting region probably come from AGN observations with ACTs, mostly the high-frequency peaked class of BL Lac objects (HBLs), which can exhibit minute-timescale variability in their VHE $\gamma$-ray fluxes, as has been shown by the HESS and MAGIC experiments in PKS 2155-3046 and Mkn 5017, respectively. These findings have given raise to considerable theoretical developments in the literature $12[13$, as existing models have to struggle to either remain simple but invoke uncomfortably large bulk Lorentz factors of 100 or higher in order to allow $\sim \mathrm{TeV} \gamma$-rays to escape from the compact area, or use multiple emitting zones, or the emission region becomes very small ${ }^{12}$. Intruigingly, the power density spectrum of one of the giant flares of PKS 2155-304 shows no sign of high-frequency cutoff in the red noise power law distribution up to the Poisson noise level, so it cannot be excluded that even 
faster variability could be detected in the light curves if they were sampled by more sensitive instruments (or if the fluxes were higher).

The BL Lac object PKS 2155-304 was also the first source of this type to be targeted during a simultaneous space-ground multi-wavelength campaign in 2009 involving Ferme ${ }^{23}$. Observing the soft spectrum of the archival EGRET measurements on this object would have been quite disconcerting since the this soft spectrum is incompatible with a smooth connection to the lowest known VHE spectrum (Fig. 31) and would therefore rule out a single radiative IC population. The Fermi observations during this campaign turned out to be actually in good agreement with the simultaneous VHE spectrum, and provided the first simultaneous IC characterization of an HBL. An interesting feature of this campaign was that the X-ray and VHE light curves, despite significant variability, were not correlated, when the VHE and optical fluxes appeared to be correlated to some degree. While the latter correlations have yet to be confirmed and understood, the observed X-ray/VHE behaviour could actually be reproduced with a simple SSC model since the highest electrons, those radiating the X-rays through the synchrotron mechanism, are barely noticed at VHE energies due to a combination of large Klein-Nishina suppression, a low density of target photons, and EBL attenuation.

With the advent of quasi-continuous, well-sampled observations of flaring BL Lac objects, VHE $\gamma$-ray light curves can now be characterized beyond finding the fastest doubling/halving time scales. Tools commonly used in X-ray observations can now usefully be applied to the whole data set instead of picking the most interesting part of it (or at least what might look as such). In this way it has now been established that, at least for PKS 2155-304, the 2006 flaring state is compatible with a stationary lognormal process ${ }^{8}$ whose power spectum density is red noise, sharing a characteristic of accreting sources shining with thermal radiation such as Cyg $\mathrm{X}-1$ or some Seyfert galaxies for instance. This might be important enough that it should be sought for not only in the other jet-dominated non-thermal emissions from AGN, but also again in the same source to find out whether the PSD slope or lognormal factor are subject to changes. Its eventual ubiquity would also have to be reproduced by time-dependent emission models.

Not much was known about BL Lac objects in the HE range, so it was not clear until Fermi started observing what exactly was happening in that regime, i.e. whether BL Lacs were just very faint, or if they were bright at higher energies where EGRET's sensitivity dropped. It was hence surprising to find many hard BL Lac objects among the bright sources seen by Ferm ${ }^{10}$. Their spectra are generally power laws over 3 decades in energy, with sometimes small deviations at energies $\geq 1 \mathrm{GeV}$. The overall SED is rather unsurprising, as the high end of the HE and the low end of the VHE spectra tend to be well within range, and in some cases even slightly overlap, since a few of the hardest sources with HE indexes of $\sim 1.5$ have a spectral bin as high as $100 \mathrm{GeV}$. A rather special case is the HBL PG 115+113, of unknown redshift, which has the largest spectral difference $(\Delta \Gamma \sim 3)$ between the HE and the VHE regimes. Since its distance is still unknown, it cannot be ruled 


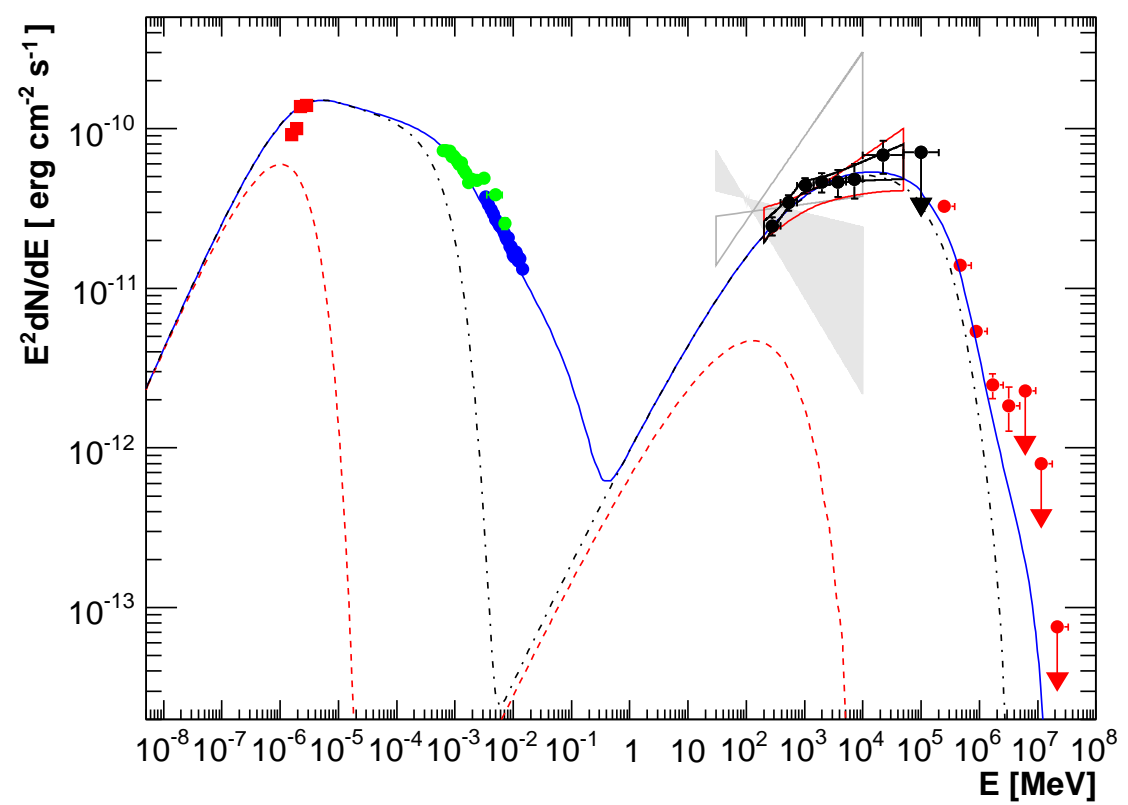

Fig. 3. The spectral energy distribution of PKS 2155-304 during the 2009 MWL campaign, involving optical measurements from ATOM, X-ray observations carried out by both Swift and RXTE, HE and VHE $\gamma$-ray observations from Fermi and HESS, respectively. The archival EGRET measurements appear in grey. The plain, dotted, and dash-dotted lines are issued from the same synchrotron self-Compton (SSC) model but with different electron cutoff energies, as described in Ref. 23 and they show that low (or quiescent) flux states can accomodate the absence of correlated $\mathrm{X}$-ray/VHE variability (see text).

out that the observed large spectral break is not related to the source but to the $\gamma$-ray propagation ${ }^{22}$. This source, like many other BL Lacs visible in both the HE and VHE ranges, exhibits surprisingly little variability in either energy band ${ }^{\sqrt{22}}$ over long time scales when it is actually known to be largely variable at X-ray and optical wavelengths.

There are high hopes that Fermi, given its intra-day overall sky surveillance and the fact that it shares the same radiative population as VHE instruments, will prove to be a more efficient provider of flaring states in blazars of interest for ACTs than all-sky X-ray monitors such as the ASM onboard Rossi-XTE or the BAT onboard Swift. But looking at the rich Astronomer's Telegram history of Fermi the very variable extragalactic sky is so far essentially composed of FSRQs and a low-frequency peaked BL Lac.

\section{3. non-blazars}

Unlike most blazars, high frequency VLBI can probe the jets in a few selected VHE sources like M87 and Centaurus $\mathrm{A}^{27}$ down to size scales 100 gravitational radii. 19 
Getting closer to localise the source of the $\gamma$-ray emission. These sources are widely supposed to be the unbeamed parent population of blazars, so even observations at large angles of the jet can provide valuable insights of jet physics. in M87, it was possible to establish that the acceleration and the collimation of the jet occurs within $\sim 100 R_{\mathrm{S}}$ (where $R_{\mathrm{S}}=2 G M / c^{2}$ is the Schwarzschild radius of the central black hole, a very important size in such systems). A joint ACT campaign, along with VLBA observations, has revealed that an increase of the nucleus radio flux might be the lagged counterpart of a similar VHE $\gamma$-ray transient, implying that the $\gamma$-ray emission, and hence the acceleration of the underlying radiative population, is likely to happen well within the collimation region 17 . Scenarios to explain how $\gamma$-ray variability can be detected in off-axis jet systems include e.g. a multiblob SSC model similar to those used for blazar emission, 24 a two-zone spine-sheath layer mechanism, 26 and pulsar-type acceleration due to centrifugally accelerated electrons in a rotating jet magnetosphere 25 Unifications of these scenarios with the beamed population is going to be an interesting development to follow in the future, as well as its long-term HE characterization by Fermi.

Another possible emerging population of $\gamma$-ray emitting AGN unseen before Fermi are the narrow-line Seyfert-1 galaxies (NLS1) of which up to now 4 have been detected $^{29}$. It was not completely unexpected that the rather rare radio-loud sample of NLS1 galaxies could be also high-energy emitters since in some scenarios they are probably have a pole-on orientation 30 . The HE spectra are however extremely steep so, given their distance, nothing is expected to be visible by ACTs by extrapolating the Fermi spectra, but suprises can arise given the rather complex SEDs which leave probably more room open to radically different models than there is for BL Lac models.

\section{Propagation effects}

\subsection{Extragalactic Background Light}

Photons of energy $E_{\gamma}$ can create an electron/positron pair when colliding with target photons of energy $E_{t}$ provided that $E_{\gamma} E_{t} \geq 2 m_{e}^{2} c^{4}$, which makes photons in the $100 \mathrm{MeV}-100 \mathrm{TeV}$ range sensitive to various existing cosmic backgrounds 31 and especially those in the far UV - far IR range (usually called the extragalactic background light, or EBL), since they have the relevant density to adversely affect the VHE extragalactic spectra when the sources are located at cosmological distances. Due to difficulties in subtracting local galactic foregrounds, this part of the cosmic background is poorly enough constrained that $\gamma$-ray attenuation can be a useful tool to probe the EBL at energies despite all the uncertainties in the intrinsic emitted spectrum. ${ }^{32}$ BL Lac objects can be used for this since the radiative particle distribution is expected to be close to the injection function, for which theoretical constraints exist, because the Lorentz factor $\gamma_{\text {cool }}$ at which electrons cool within the dissipative region is expected to be large enough $\frac{21}{1}$ that the radiative electron spectrum is not affected within a time $\sim R / c$. The use of such constraints turn 


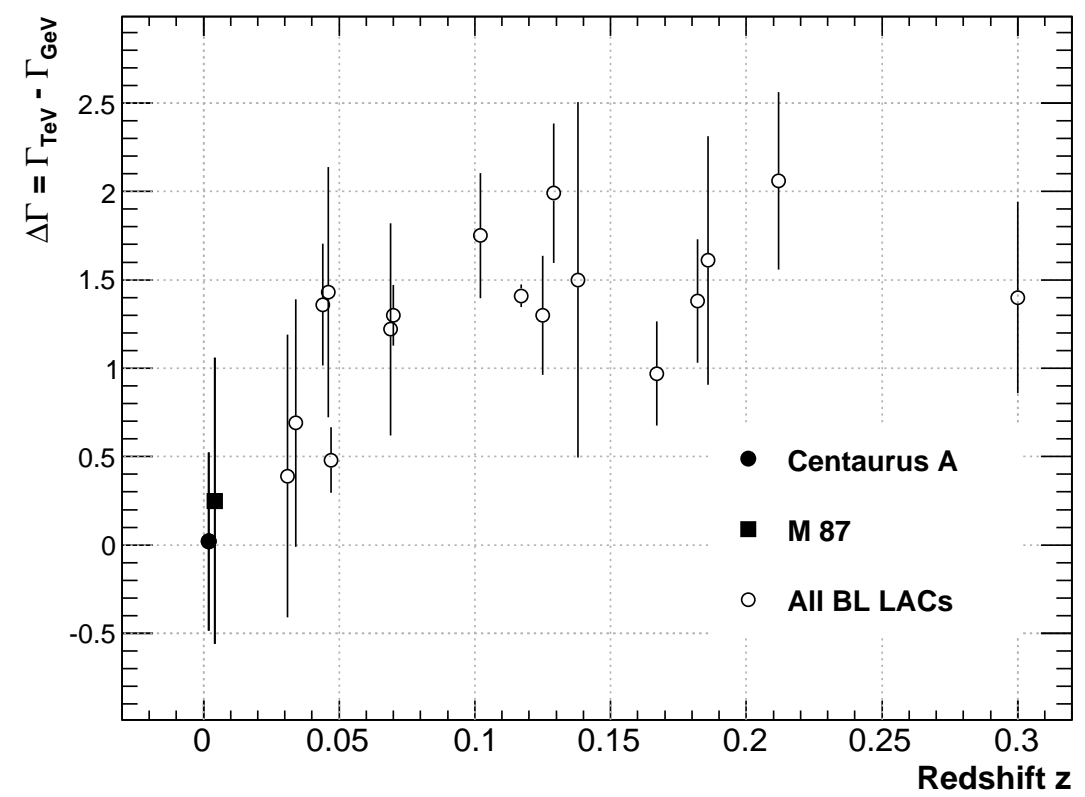

Fig. 4. The evolution of the difference between HE and VHE photon indexes (Ref. 4), showing that the spectral change becomes increasingly more important the more distant the observed source is.

out to be so far the most efficient way to constrain the EBL and hence properties of star formation rate and early Population III stars 32 34 The FSRQ class, despite the advantage of being at larger distances on average than BL Lacs, might be more tricky to use for such purposes, since not only much faster cooling time scales deviate the radiative spectrum faster from the injected one, but also because significant $\gamma$ ray absorption is expected inside the source itself 20 . Another original use of EBL attenuation is the possibility to constrain the Hubble constant 28 .

It seems however that, at least for distant BL Lacs $(z \geq 0.2)$, most of the VHE spectra contain photons up to hundreds of $\mathrm{GeV}$, but rarely up to $\mathrm{TeV}$ energies. Interestingly, extending the Fermi spectrum by (just) one decade, along with a conservative EBL attenuation model, reproduces the VHE spectra quite well. This could mean that, for most of those hard Fermi sources, the observed SED peak is not necessarily the intrinsic luminosity peak, which could be in some cases be located at energies well above $1 \mathrm{TeV}$. This can be seen in Figure4, where a clear evolution of the observed spectral break with the redshift is possibly a model-independent signature of the increasing effect of EBL attenuation, further complicating the characterization of the intrinsic emission. 


\subsection{Time-of-flight measurements}

If it was well known that high-energy $\gamma$-rays can be an interesting probe of the contents of the space they travel through, that they can also be a probe of space itself is a rather new and at least as interesting development. It appears that some models for quantum gravity predict an energy-dependence on the speed of light through Lorentz invariance violation, $\frac{35]}{3}$ which can be probed when high-energy gamma-rays travel over cosmological distances. This has in turn been searched for by quantifying possible energy-dependent lags in AGN light curves (assuming no intrinsic timeeffect cause), which has provided some of the most constraining upper limits to the energy scale at which this happens (for a review see, e.g., Ref. [36).

\section{Prospectives}

Besides high expectations for the coming years of Fermi results, The ACT systems have also upgrading plans which will improve the VHE knowledge of the sky. The MAGIC collaboration is now operating a second large telescope, and the HESS collaboration is building a central large telescope which will operate with the 4 smaller ones. A major change will come from the construction of larger Cherenkov observatories such as the Cherenkov Telescope Array (CTA) or the Advanced gamma-ray Imaging System (AGIS), with sensitivities improving by an order of magnitude at $\sim 1 \mathrm{TeV}$ and a lower energy threshold. However, in the most optimistic case, this is not likely to happen before the next Marcel Grossmann meeting, which will be a more appropriate venue to discuss these developments in further details.

\section{References}

1. A. Abdo et al., The Astrophysical Journal 7081254 (2010)

2. A. Abdo et al., The Astrophysical Journal, in preparation (first-year catalog paper)

3. G. Tosti, these proceedings

4. A. Abdo et al., The Astrophysical Journal, submitted (arXiv:1002.0150)

5. G. Nandikotkur et al., The Astrophysical Journal 657706 (2007)

6. F. Aharonian et al., The Astrophysical Journal 664, 71 (2007)

7. J. Albert et al., The Astrophysical Journal 669862 (2007)

8. B. Degrange et al., PoS(BLAZARS2008) 63, 16 (2008)

9. A. Abdo et al., The Astrophysical Journal 700127 (2009)

10. A. Abdo et al., The Astrophysical Journal 7071310 (2009)

11. R. D. Blandford and R. L. Znajek, Monthly Notices of the Royal Astronomical Society 179, 433 (1977)

12. A. Levinson, these proceedings

13. H. Sol, these proceedings

14. L. Escande and Y. T. Tanaka, The Astronomer's Telegram 2328

15. G. Ghisellini et al., Monthly Notices of the Royal Astronomical Society 301451 (1998)

16. G. Fossati et al., Monthly Notices of the Royal Astronomical Society 299433 (1998)

17. V. A. Acciari et al., Science 3255939 (2009)

18. J. Albert et al., Science 3201752 (2008)

19. M. Georganopoulos, these proceedings 
20. F. Aharonian, L. Costamante, and D. Khangulyain Monthly Notices of the Royal Astronomical Society 3871206 (2008)

21. G. Ghisellini, F. Tavecchio, et al., Monthly Notices of the Royal Astronomical Society 402497 (2010)

22. A. Abdo et al., The Astrophysical Journal 7081310 (2009)

23. F. Aharonian et al., The Astrophysical JournalLetters 696150 (2009)

24. J.-P. Lenain, C. Boisson, H. Sol, and K. Katarzynski, Astronomy and Astrophysics 478111 (2008)

25. F. Rieger and F. A. Aharonian, Astronomy and Astrophysics 4795 (2008)

26. F. Tavecchio and G. Ghisellini Monthly Notices of the Royal Astronomical Society $\mathbf{3 8 5}$ $98(2008)$

27. F. Aharonian et al., The Astrophysical Journal 69540 (2009)

28. A. Gorecki, these proceedings

29. A. Abdo et al., The Astrophysical Journal 707142 (2009)

30. R. Remillard et al.,Nature 350589 (1991)

31. M. T. Ressell and M. J. Turner, Comments on Astrophysics 14323 (1990)

32. F. Aharonian et al., Nature 4401018 (2006)

33. P. Coppi and F. Aharonian, Astroparticle Physics 1135 (1999)

34. M. Raue, T. Kneiske and D. Mazin, Astronomy and Astrophysics 49825 (2009)

35. G. Amelino-Camelia, J. Ellis, N. E. Mavromatos, D. V. Nanopoulos and S. Sarkar, Nature 395525 (1998)

36. R. Wagner, AIP Conference Proceedings 1112187 (2009) 